\title{
The kinematics of the diffuse ionized gas in NGC 4666
}

\author{
P. Voigtländer ${ }^{1}$, P. Kamphuis ${ }^{1,2}$, M. Marcelin ${ }^{3}$, D. J. Bomans ${ }^{1}$, and R.-J. Dettmar ${ }^{1}$ \\ 1 Astronomisches Institut, Ruhr-Universität Bochum, Universitätsstr. 150, 44801 Bochum, Germany \\ e-mail: voigtlaender@astro.rub.de \\ 2 CSIRO Astronomy \& Space Science, PO Box 76, Epping, NSW 1710, Australia \\ 3 Aix-Marseille Université, CNRS, LAM (Laboratoire d'Astrophysique de Marseille) UMR 7326, 13388 Marseille, France
}

Received 25 January 2013 / Accepted 11 April 2013

\section{ABSTRACT}

\begin{abstract}
Context. The global properties of the interstellar medium with processes such as infall and outflow of gas and a large scale circulation of matter and its consequences for star formation and chemical enrichment are important for the understanding of galaxy evolution. Aims. In this paper we studied the kinematics and morphology of the diffuse ionized gas (DIG) in the disk and in the halo of the star forming spiral galaxy NGC 4666 to derive information about its kinematical properties. Especially, we searched for infalling and outflowing ionized gas.

Methods. We determined surface brightness, radial velocity, and velocity dispersion of the warm ionized gas via high spectral resolution $(R \approx 9000)$ Fabry-Pérot interferometry. This allows the determination of the global velocity field and the detection of local deviations from this velocity field. We calculated models of the DIG distribution and its kinematics for comparison with the measured data. In this way we determined fundamental parameters such as the inclination and the scale height of NGC 4666, and established the need for an additional gas component to fit our observed data.

Results. We found individual areas, especially along the minor axis, with gas components reaching into the halo which we interpret as an outflowing component of the DIG. As the main result of our study, we were able to determine that the vertical structure of the DIG distribution in NGC 4666 is best modeled with two components of ionized gas, a thick and a thin disk with $0.8 \mathrm{kpc}$ and $0.2 \mathrm{kpc}$ scale height, respectively. Therefore, the enhanced star formation in NGC 4666 drives an outflow and also maintains a thick ionized gas layer reminiscent of the Reynold's layer in the Milky Way.
\end{abstract}

Key words. galaxies: kinematics and dynamics - galaxies: halos - galaxies: evolution - galaxies: individual: NGC 4666 techniques: radial velocities

\section{Introduction}

It is well known that stellar winds and supernovae explosions are the processes that contaminate the interstellar medium (ISM) with heavy elements (Shields 2002). Furthermore, supernova explosions can trigger additional star formation because of the pressure they provide to the ISM. On galactic scales stellar winds and supernova explosions can influence the galactic evolution in an additional way. They locally heat and accelerate the ISM, thus creating so-called superbubbles (SBs; Tomisaka et al. 1981). Because of lower pressures in the vertical direction, these SBs expand faster in the vertical direction creating structures, much like smoking chimneys, through which the gas is able to flow into the halo of the galaxy (Norman \& Ikeuchi 1989).

Depending on the energy of this outflow, one can distinguish two scenarios. Either the gas has enough energy to escape the host galaxy, creating a galactic wind that enriches the intergalactic medium (IGM; Heckman 2002; Veilleux et al. 2005), or the gas is unable to escape the gravitational potential of the host galaxy. In the second case the chemical enrichment of the galaxy is delayed and a large gaseous halo can be created around the galaxy.

Shapiro \& Field (1976) described the processes of the gas dynamics into the halo and the fall-back as a galactic fountain. In this model the ejected gas condenses to clouds in the halo and falls back onto the disk. Recently, Marinacci et al. (2011) studied the galactic fountain model by using two-dimensional hydrodynamical simulations of a cloud ejected from the disk into the hot coronal gas. They described the influence of radiative cooling on the structure of this cloud and detected in their simulations a deceleration of the ejected cloud as a function of the height above the plane, i.e., a vertical velocity gradient. This is also called a lagging halo. Lagging halos are observed in several galaxies e.g., NGC 5775 (Heald et al. 2006b) or NGC 891 (Fraternali et al. 2005; Heald et al. 2006a; Kamphuis et al. 2007; Oosterloo et al. 2007).

The ejected gas in the halo exists in different phases. This paper is focused on the extraplanar diffuse ionized gas (eDIG). This extraplanar component of the diffuse ionized gas (DIG) was detected in several galaxies, for example in our Milky Way (Reynolds 1990). The study of the eDIG in other galaxies has been made in several galaxies in $\mathrm{H} \alpha$ (Dettmar 1990; Rossa \& Dettmar 2000, 2003a,b; Rand et al. 1990; Rand 1996). Detailed kinematic studies of the halo gas were made in several dwarf galaxies (Martin et al. 2002; van Eymeren et al. 2009a,b) and continued in this paper for the SABc galaxy NGC 4666.

Here we present a study of the kinematics of the DIG in the disk as well as in the halo of NGC 4666 using Fabry-Pérot interferometry. In this context we will determine the possible consequences of the strong starburst. This includes the detection of outflows, described by Dahlem et al. (1997), and the physical properties of an eDIG layer (thick disk).

The galaxy NGC 4666 supposedly has a high star formation rate (SFR; Walter et al. 2004) of 7.0 $M_{\odot} \mathrm{yr}^{-1}$ (Dahlem et al. 1997). It has been well studied at several wavelengths, e.g., 
Table 1. General parameters of NGC 4666.

\begin{tabular}{|c|c|}
\hline Parameters (Unit) & Value \\
\hline \multicolumn{2}{|l|}{ optical center: } \\
\hline$\alpha(\mathrm{J} 2000.0)^{a}$ & $12^{\mathrm{h}} 45^{\mathrm{m}} 08^{\mathrm{s}} .6$ \\
\hline$\delta(\mathrm{J} 2000.0)^{a}$ & $-00^{\circ} 27^{\prime} 43^{\prime \prime}$ \\
\hline$D[\mathrm{Mpc}]^{a}$ & 18.225 \\
\hline Helioc. Radial velocity $\left(\mathrm{km} \mathrm{s}^{-1}\right)^{a}$ & 1529 \\
\hline Galaxy type $^{a}$ & $\mathrm{SABc}$ \\
\hline Size $\left(\right.$ Maj. $\times$ Min. Diameter) $\left({ }^{\prime}\right)^{a}$ & $4.6 \times 1.3$ \\
\hline Inclination $\left({ }^{\circ}\right)^{b}$ & 69.6 \\
\hline Position angle $\left({ }^{\circ}\right)^{c}$ & 42 \\
\hline Max. rotation velocity $\left(\mathrm{km} \mathrm{s}^{-1}\right)^{b}$ & $192.9 \pm 2.3$ \\
\hline
\end{tabular}

Notes. ${ }^{(a)}$ Data from NASA/IPAC Extragalactic Database (NED) (http://ned.ipac. caltech. edu/). ${ }^{(b)}$ Data from the Lyon/Meudon Extragalactic Database (LEDA) (http://leda.univ-lyon1.fr/). (c) Data from Springob et al. (2007).

in $\mathrm{H} \alpha$ and $R$-band (Lehnert \& Heckman 1995, 1996a,b), radio continuum and soft X-ray (Dahlem et al. 1997, 1998), and $21 \mathrm{~cm}$ and CO (Walter et al. 2004).

Garcia (1993) determined NGC 4666 to be a member of a small group of galaxies. Besides NGC 4666 this group is composed of NGC 4632 and NGC 4668. In this context it is currently believed that the strong starburst is triggered by ongoing far-field gravitational interactions (Walter et al. 2004). Table 1 shows the general parameters of the galaxy.

This paper is structured as follows: in Sect. 2 we describe the observations and the data reduction and in Sect. 3 follows the results of our analysis of the galaxy. In Sect. 4 our $\mathrm{H} \alpha$ observations are compared to other observations, especially in other wavelengths, and in Sect. 5 we summarize our results.

\section{Observations and data reduction}

For our observations of NGC 4666 we used the high spectral resolution $(R \approx 9000)$ Marseille Fabry-Pérot interferometer (FPI) on the $1.93 \mathrm{~m}$ telescope at the Observatoire de HauteProvence (OHP) in France. The FPI was equipped with a photon counting system (Gach et al. 2002) which provides a field of view (FOV) of $5.4 \times 5.4 \operatorname{arcmin}^{2}$ on $512 \times 512$ pixels $^{2}$. This results in a pixel size of $0.63^{\prime \prime}$. Furthermore, we used a narrow band $\mathrm{H} \alpha$ filter with a width of $10.10 \AA$ and a maximum transmission of $70 \%$ at $6597.7 \AA$. The systemic velocity of the galaxy is $1529 \mathrm{~km} \mathrm{~s}^{-1}$ (see Table 1) which redshifts the $\mathrm{H} \alpha$ line to $6596.3 \AA$, close to the maximum transmission of the chosen $\mathrm{H} \alpha$ filter. The free spectral range of the FPI is about $8.34 \AA$ or $379 \mathrm{~km} \mathrm{~s}^{-1}$. This results, using a 32-channel mode, in a sampling step of $0.26 \AA$ or $11.83 \mathrm{~km} \mathrm{~s}^{-1}$, respectively. By measuring the FWHM of a neon line, emitted by a neon calibration lamp, the finesse could be determined to 11.5 and the spectral resolution to $0.73 \AA$ or $33.0 \mathrm{~km} \mathrm{~s}^{-1}$, which is less than 3 channels.

Because of reflections within the FPI, a ghost image of the galaxy is produced. This ghost is mirrored in the center of the FOV. To prevent an overlap of the real signal and the signal of the ghost we positioned the galaxy about $1^{\prime}$ offset from the center of the FOV. This offset is small enough that the galaxy does not leave the FOV, but is large enough to ensure a clear separation between the ghost and the galaxy, thus preventing any contamination.
The observations were obtained in two nights in early February 2011. In these two nights the galaxy was observed in 50 and 47 cycles, respectively. A failure in the cooling system increased noise and created bad cycles, which were then removed, leaving us with 50 and 44 cycles for each night. To identify possible extended halo structures we moved the telescope for the second observation about $1.7^{\prime}$ west in order to position the ghost image once east and once west of the galaxy. With an integration time of $10 \mathrm{~s}$ per channel in one cycle we reached a total integration of $30080 \mathrm{~s}(\approx 8.36 \mathrm{~h})$ or $940 \mathrm{~s}(\approx 15.7 \mathrm{~min})$ per channel.

The data were reduced with the IDL program FPreduc (Daigle et al. 2006; Epinat 2009). Both cubes were reduced independently. In order to correct for fluctuations in the telescope positioning, the position of every $10 \mathrm{~s}$ exposure was corrected by matching the positions of several stars in the FOV. Furthermore, the sky lines were subtracted and a heliocentric velocity correction was applied. With the koords routine of the karma package (Gooch 1996) both cubes were provided with a coordinate system by matching four stars in the FOV to their counterparts in a Digitized Sky Survey (DSS) image.

After the reduction with FPreduc the continuum was still visible in the data. Therefore, the continuum was subtracted using the continuum image which was produced during the data reduction. Once both cubes were fully reduced they were moved to the same pixel coordinates and combined.

The observed velocities of NGC 4666 cover a larger range than the available free spectral range. Therefore, some areas with extreme velocities appear in channels that are offset from their true velocities by the spectral range. These velocities are measured from the previous or next order of the FPI. In order to correct this, the cube was extended with 16 channels, 10 channels in front and 6 channels behind the normal velocity range. Such parts of emission which contained velocities of the previous order were added in the channels behind the normal range and such parts of emission which contained velocities of the next order were added in front of the normal range. Furthermore, these regions were blanked within the normal range.

The subsequent analysis of the data cube was done with the GIPSY package (van der Hulst et al. 1992). Although GIPSY is mainly used for the analysis of interferometric observations of $\mathrm{H}_{\mathrm{I}}$ line emission, it has many functions that can also be applied to FPI observations of $\mathrm{H} \alpha$ emission. To optimize the signal-tonoise ratio $(\mathrm{S} / \mathrm{N})$ the cube was spatially smoothed by using the task SMOOTH, from 3.6", which is equal to the average seeing, to a size of $5.0^{\prime \prime}$. This value of $5.0^{\prime \prime}$ was determined to be the best compromise between increasing the $\mathrm{S} / \mathrm{N}$ in the outer parts of the galaxy and a good spatial resolution. For the following we define the number of complete pixels within a block of $5.0^{\prime \prime} \times 5.0^{\prime \prime}$ as one resolution element, which corresponds to $7 \times 7$ pixels.

\section{Analysis}

In our analysis we focus on five different areas. Initially, we determine the sensitivity of our observation, then we verify the statement of (Walter et al. 2004) that NGC 4666 is a starburst galaxy by determining the SFR. The third step is the velocity analysis of our data. From this analysis we obtain a rotation curve for NGC 4666 and a good impression of the kinematics. In order to understand how much of the gas is in the disk and how much is in the halo, we use this kinematical information to construct a model for the disk. Finally, we compare our disk model to the data to understand structures in the halo of the galaxy. 


\subsection{Sensitivity of our observation and verification of the SFR}

To determine the sensitivity of our data we added all channels to generate an integrated $\mathrm{H} \alpha$ map which we will refer to as the intensity map from here on. With a total $\mathrm{H} \alpha$ flux of $4.7 \times 10^{-12} \mathrm{erg} \mathrm{s}^{-1} \mathrm{~cm}^{-2}$ of NGC 4666, determined by Moustakas \& Kennicutt (2006) with drift-scanning spectroscopy, we determined the $3 \sigma$ sensitivity of our observations to be $5.6 \times 10^{-18} \mathrm{erg} \mathrm{s}^{-1} \mathrm{~cm}^{-2} \operatorname{arcsec}^{-2}$. Another common unit to describe the intensity of emission is the so-called emission measure $(\mathrm{EM})$. It is given by

$E M=\int n_{\mathrm{e}}^{2} \mathrm{~d} r$

where $n_{\mathrm{e}}$ is the electron density and $r$ the length along the line-of-sight. For a direct calculation we could use the formula (Dettmar 1992)

$E M=5 \times 10^{17} F_{\mathrm{H} \alpha} / \Omega \mathrm{cm}^{-6} \mathrm{pc}$,

with the measured flux $F_{\mathrm{H} \alpha}$ in $\operatorname{erg~cm}^{-2} \mathrm{~s}^{-1}$ and $\Omega$ the collecting area in $\square^{\prime \prime}$. This results in an emission measure of $E M=2.8 \mathrm{~cm}^{-6} \mathrm{pc}$ for the aforementioned sensitivity.

NGC 4666 is described as a superwind galaxy with a high SFR of $7.0 M_{\odot} \mathrm{yr}^{-1}$ (Dahlem et al. 1997) or even as a starburst galaxy (Walter et al. 2004). This value should also be verified for our discussion. For $\mathrm{H} \alpha$ we took the aforementioned flux of $4.7 \times 10^{-12} \mathrm{erg} \mathrm{s}^{-1} \mathrm{~cm}^{-2}$ and for comparison the flux density in FIR at $60 \mu \mathrm{m}$ of $37.34 \mathrm{Jy}$ (Soifer et al. 1989). The $\mathrm{H} \alpha$ flux is influenced by extinction. To obtain the extinction, we used the $\mathrm{H} \beta$ flux of $0.90054 \times 10^{-12} \mathrm{erg} \mathrm{s}^{-1} \mathrm{~cm}^{-2}$ determined by Kennicutt et al. (2009), and calculated a Balmer decrement of $\frac{\mathrm{H} \alpha}{\mathrm{H} \beta}=5.22$ which is above the expected ratio of 2.86 for a temperature of $10000 \mathrm{~K}$ (Osterbrock 1989). Taking into account the reddening law (Fitzpatrick 1999), the formula for the color excess $E(B-V)$ is given by Xiao et al. (2012) as

$E(B-V)=1.99 \times \log \frac{\mathrm{H} \alpha / \mathrm{H} \beta}{2.86}$.

This results in a color excess of $E(B-V)=0.52$ which corresponds to $A(V)=1.61$, with $A(V)=3.1 \times E(B-V)$, and $A(B)=2.13$. With $A(\mathrm{H} \alpha)=0.538 \times A(B)$ the extinction of the $\mathrm{H} \alpha$ line can be calculated as $A(\mathrm{H} \alpha)=1.15$. Therefore, the total $\mathrm{H} \alpha$ flux is increased by a factor of 2.88 to $13.51 \times$ $10^{-12} \mathrm{erg} \mathrm{s}^{-1} \mathrm{~cm}^{-2}$. For the additional calculations, we used the mean distance of $18.2 \mathrm{Mpc}$, provided in $\mathrm{NED}^{1}$, whereas Dahlem et al. (1997) used 26.3 Mpc. The different values given by NED are mostly obtained by the Tully-Fisher relation and are approximately $19 \mathrm{Mpc}$. The final value of NED we used is the average of all these single values. In contrast, the value of Dahlem et al. (1997) is based on the redshift with a correction for the Virgo infall. Since both methods are not very accurate we prefer to take the average from NED. Using the formulas of Kennicutt (1998), we obtained an SFR of

$S F R=7.9 \times 10^{-42} L_{\mathrm{H} \alpha} \frac{M_{\odot} \mathrm{yr}^{-1}}{\mathrm{erg} \mathrm{s}^{-1}}=4.24 M_{\odot} \mathrm{yr}^{-1}$

for $\mathrm{H} \alpha$ and

$S F R=4.5 \times 10^{-44} L_{\mathrm{FIR}} \frac{M_{\odot} \mathrm{yr}^{-1}}{\mathrm{erg} \mathrm{s}^{-1}}=2.2 M_{\odot} \mathrm{yr}^{-1}$

for far-infrared (FIR).

1 NASA Extragalactic Database, http://ned.ipac.caltech.edu/
Using the distance of 26.3 Mpc (Dahlem et al. 1997) the SFRs would increase by a factor of about 2 . This results in a good agreement between our $\mathrm{H} \alpha \mathrm{SFR}$ and value of $7.0 M_{\odot} \mathrm{yr}^{-1}$ determined by Dahlem et al. (1997). The remaining differences between the SFR in $\mathrm{H} \alpha$ and FIR can easily be explained by galaxy specific characteristics (Kennicutt 1998). In this context Kennicutt (1998) referenced Buat \& Xu (1996) who determined a coefficient of $8_{-3}^{+8} \times 10^{-44}$ instead of the abovementioned $4.5 \times 10^{-44}$.

\subsection{Kinematic analysis}

Before starting the further analysis of the data cube the abovementioned ghost images of the galaxy below and above the disk were removed. For the further analysis the GIPSY task XGAUFIT was used. First a single Gaussian fit of every pixel with XGAUFIT was performed by using blocking filters for velocity and dispersion/linewidth ${ }^{2}$. For the velocity only values within the velocity range of the extended cube were accepted. For the linewidth the minimum value was set to the instrumental dispersion of $14 \mathrm{~km} \mathrm{~s}^{-1}$, which follows directly from the abovementioned spectral resolution of the instrument. The maximal linewidth was set to $162 \mathrm{~km} \mathrm{~s}^{-1}$, which is equal to the dispersion of a Gaussian with a FWHM of the free spectral range. All these filters suppress unphysical fitting results. The intensity map was masked at the $3 \sigma$ level and subsequently used as a mask for the velocity field. As a last processing step a filter that blanked any isolated pixels was applied to the data. This results in a wellcleaned velocity field which can be seen in Fig. 1. All these steps were also done for the linewidth map which was produced with XGAUFIT, resulting in Fig. 2 (center). In both figures the black contours describe the intensity at $3 \sigma, 6 \sigma, 12 \sigma, 24 \sigma$, etc.

Because of our predominant interest in the vertical structure, the velocity map and the linewidth map were rotated so that the major axis of the galaxy is parallel to the $x$-axis. Moreover, the global coordinate system in right ascension and declination was replaced by a relative coordinate system that describes the radial and vertical offset to the center of NGC 4666.

These maps of NGC 4666 show a lopsidedness with a radial extent of the approaching part of about $110^{\prime \prime}$ and a radial extent of the receding part of about $125^{\prime \prime}$. Furthermore, the velocity map immediately shows several features beyond this lopsidedness. When comparing the intensity contours in the velocity map, one can see the influence of the H II regions to the measured velocities, e.g., at the $\mathrm{H}_{\text {II }}$ regions at a radial/vertical offset of $-74^{\prime \prime} / 16^{\prime \prime}$ and $-30^{\prime \prime} / 8^{\prime \prime}$.

To determine the position angle and kinematical center and to obtain a rotation curve for NGC 4666, a tilted ring model was fitted by means of $\chi^{2}$ minimization with the GIPSY task ROTCUR on the velocity map. Because of the lopsidedness of NGC 4666, only the inner $105^{\prime \prime}$ could be fitted well. The width of the rings was set to $5^{\prime \prime}$, our spatial resolution after smoothing. In a first run the position of the center of the galaxy, the rotation velocity, the systemic velocity, the inclination, and the position angle were all fitted in order to obtain initial estimates. Step-by-step single parameters were fixed using the average value over all the rings to improve the fit for the other parameters. In the last iteration only the rotation velocity and the inclination were not fixed. The results are shown in Figs. 3 and 4 for the rotation curve and the inclination, respectively. All the other parameters and the average of the inclination are given in Table 2 .

\footnotetext{
2 We define the linewidth as the dispersion of the Gaussian fit of the data which is not corrected for the instrumental dispersion.
} 


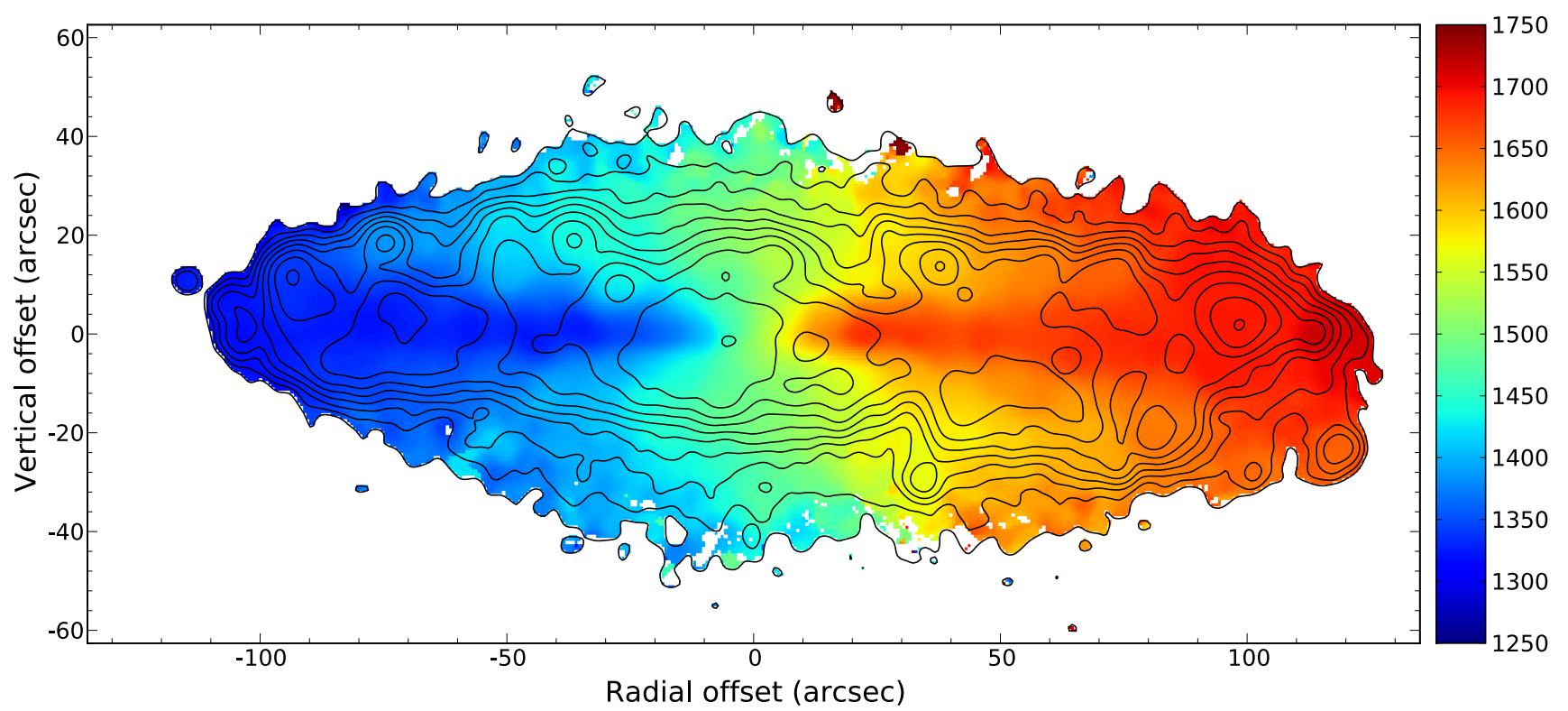

Fig. 1. Velocity map of NGC 4666 with coordinates in offsets from the center of the galaxy in arcseconds. The contours show the intensity map at $3 \sigma, 6 \sigma, 12 \sigma, 24 \sigma$, etc.

Looking at Fig. 3 one can see an almost flat rotation curve with a maximal rotation velocity of about $190-195 \mathrm{~km} \mathrm{~s}^{-1}$ which agrees well with the value found in the literature given in Table 1. The position of the galactic center and the position angle also correspond to the values found in the literature (see Table 1). The value of the position angle shows this offset of about $180^{\circ}$ because of the definition in ROTCUR; in ROTCUR the position angle is defined as the counterclockwise angle between the north direction and the major axis of the receding part of the galaxy. The systemic velocity, the inclination and the position angle are the average values over all the rings up to $105^{\prime \prime}$ with the standard deviation as the error.

In the next step, the velocity offset along the minor axis of up to $100 \mathrm{~km} \mathrm{~s}^{-1}$ described by Dahlem et al. (1997) will be investigated. To verify this in our data we determined the velocity for every resolution element along the minor axis. Thus, we obtained for every vertical offset $y$ along the minor axis a $\Delta v$ which is defined as $\Delta v=v_{y}-v_{0}$, with $v_{y}$ as the velocity at a given vertical offset and $v_{0}$ as the velocity of the central resolution element $\left(1510 \mathrm{~km} \mathrm{~s}^{-1}\right)$. In Fig. 5 we plot $\Delta v$ vs. the vertical offset of our data in comparison to the data of Dahlem et al. (1997). To do this, we switched the sign of the vertical offset in the data of Dahlem et al. (1997) because of the $180^{\circ}$ difference in the assumed PA.

In direct comparison, the general shape of the velocities between $-20^{\prime \prime}$ and $+20^{\prime \prime}$ vertical offset agrees well with the one presented in Dahlem et al. (1997). The velocity variations in the Dahlem et al. (1997) data and in our data in this inner area can be explained by the influence of $\mathrm{H}_{\mathrm{II}}$ regions. The differences in the outer part are probably a combined effect of reduced $\mathrm{S} / \mathrm{N}$ and a difference in velocity resolution $\left(\lambda_{\text {res }}=0.73 \AA \widehat{\equiv} 33 \mathrm{~km} \mathrm{~s}^{-1}\right.$ in this work, $\lambda_{\text {res }}=4 \AA \widehat{\equiv} 182 \mathrm{~km} \mathrm{~s}^{-1}$ in Dahlem et al. 1997).

To study further this velocity offset in the outer regions and to detect other anomalies in the kinematics of the galaxy, the linewidth of the Gaussian fit is a useful tool. Therefore, we have chosen six areas to describe features in this linewidth map in more detail. The profiles of these regions can be seen in Fig. 2 around the linewidth map. First, the general linewidth is about $30-35 \mathrm{~km} \mathrm{~s}^{-1}$, shown by way of example at position 1 . The profile can be well fitted by one Gaussian (red line). Where we have compact $\mathrm{H}$ II regions (black contour lines) the dispersion is about $30 \mathrm{~km} \mathrm{~s}^{-1}$ or lower. In this linewidth map we see a region of large linewidth at a vertical offset of about $30^{\prime \prime}$ along the minor axis (position 2). The profile at this position shows a double Gaussian structure with a smaller Gaussian with a central position at $1421 \mathrm{~km} \mathrm{~s}^{-1}$ and a bigger one at $1517 \mathrm{~km} \mathrm{~s}^{-1}$ (green lines), which is in good agreement with our central velocity, and also in better agreement with Dahlem et al. (1997) for this vertical offset (see Fig. 5). By fitting merely one Gaussian to the line profile our central position is shifted to a lower value due to this first component at $1421 \mathrm{~km} \mathrm{~s}^{-1}$. Without that component the velocity keeps roughly constant for a positive vertical offset. This would be expected for a normal flat rotating galaxy. The first component with a velocity of about $100 \mathrm{~km} \mathrm{~s}^{-1}$ below the systemic velocity can be interpreted as an outflowing component of the gas.

There are several other conspicuous areas in the linewidth map. In the center there is an $x$-shaped region with a larger linewidth which also shows a double Gaussian structure (e.g., at position 3). The linewidth map shows a linewidth for this structure up to $60-80 \mathrm{~km} \mathrm{~s}^{-1}$. Furthermore, there is a significant rise of the linewidth, up to $50 \mathrm{~km} \mathrm{~s}^{-1}$, in the outer part of the galaxy above $20^{\prime \prime}$ and below $-20^{\prime \prime}$ vertical offset. In the half of the galaxy with a negative radial offset the linewidth rises even up to $130 \mathrm{~km} \mathrm{~s}^{-1}$, e.g., at position 4 . Within these regions of large linewidths is a small hook-like structure where the linewidth drops down to $30-40 \mathrm{~km} \mathrm{~s}^{-1}$, marked as position 5. A detailed view of this position shows a similar shape as position 4 , but here the amplitude of the first component, at $1335 \mathrm{~km} \mathrm{~s}^{-1}$, is significantly lower than the second component at $1427 \mathrm{~km} \mathrm{~s}^{-1}$ and close to the noise level. Therefore, the fit was dominated by the second component which has a small linewidth. Moreover, there is a region similar to position 2 where the linewidth rises within a compact area, marked as position 6 in Fig. 2. Both areas, 5 and 6, will be discussed in more detail later in comparison to another independent $\mathrm{H} \alpha$ observation. 

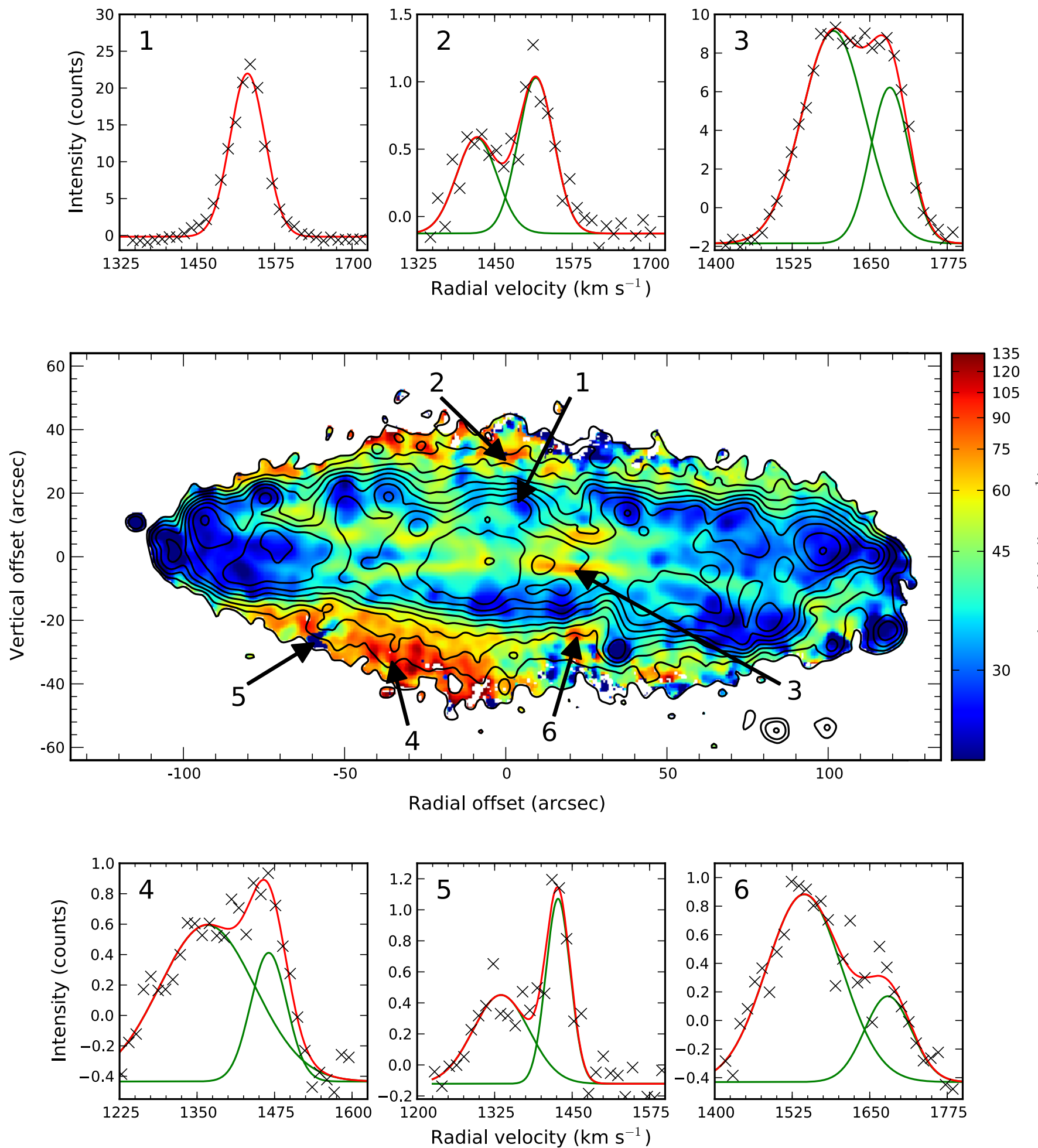

Fig. 2. Linewidth map of NGC 4666, resulting from a single Gaussian fit, with coordinates given in distances in arcseconds to the center of the galaxy. The contours show the intensity map at $3 \sigma, 6 \sigma, 12 \sigma, 24 \sigma$, etc. Furthermore, some profiles of chosen pixels are displayed to show that high linewidths are double Gaussian profiles in the selected areas. When there are two Gaussians, the single Gaussian profiles are displayed in green and the sum of both in red.

The negative baselines of some profiles are a result of oversubtracted sky lines and because the continuum subtraction was optimized for the galactic center. Because of the oversubtraction, the above-mentioned calculated sensitivity is just an upper limit, but it does not affect the further profile analysis because it is merely a shift along the intensity axis and does not influence the shape of the profile. To understand the origin of all these double Gaussians we constructed a set of models to compare with the data. We will describe these models in the following section. 


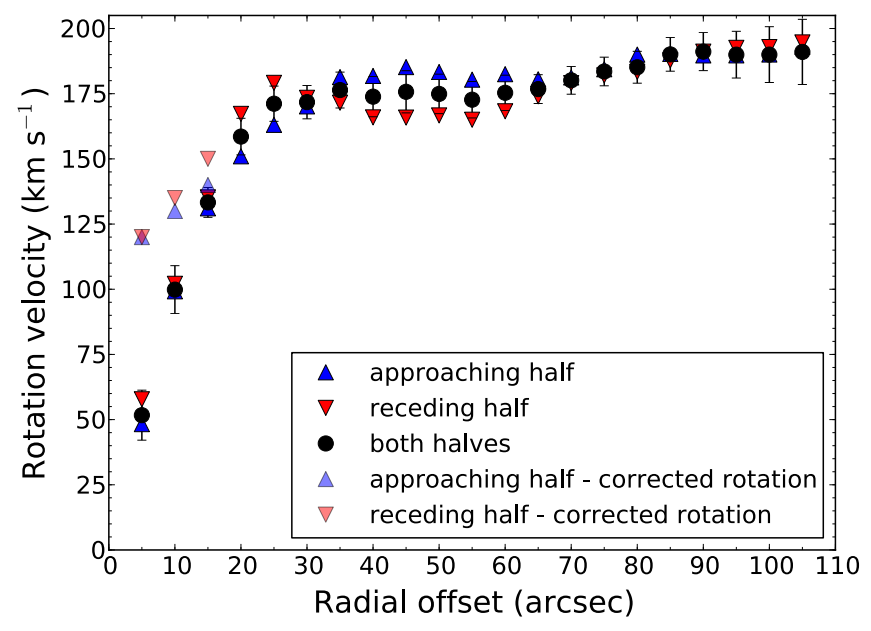

Fig. 3. Rotation velocity vs. radial distance from the center. The blue triangles describe the approaching half of the galaxy, the red triangles the receding half, and the black dots both halves in a common calculation with the sigma of the velocity given by ROTCUR as error bars. The semitransparent triangles in blue and red describe the corrected rotation of the approaching and the receding half for the final model, respectively.

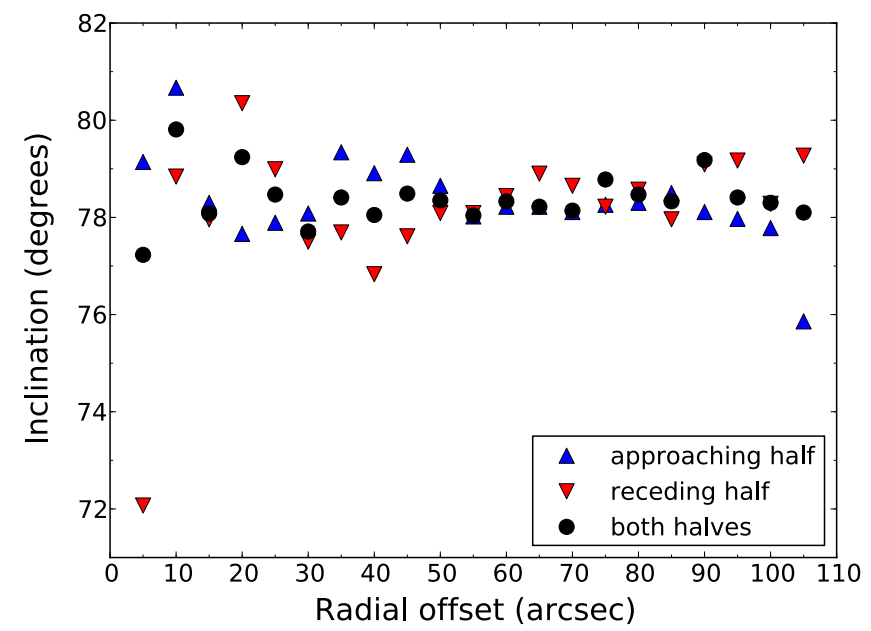

Fig. 4. Inclination vs. radial distance from the center. Symbols as in Fig. 3.

Table 2. Results of ROTCUR for NGC 4666.

\begin{tabular}{ll}
\hline \hline Parameters (Unit) & Value \\
\hline kinematical center: & \\
$\alpha(\mathrm{J} 2000.0)$ & $12^{\mathrm{h}} 45^{\mathrm{m}} 08^{\mathrm{s}} 6.8$ \\
$\delta(\mathrm{J} 2000.0)$ & $-00^{\circ} 27^{\prime} 43.9^{\prime \prime}$ \\
Systemic velocity $\left(\mathrm{km} \mathrm{s}^{-1}\right)$ & $1506 \pm 4$ \\
Inclination $\left({ }^{\circ}\right)$ & $78.3 \pm 0.5$ \\
Position angle $\left(^{\circ}\right)$ & $222.6 \pm 0.8$ \\
\hline
\end{tabular}

Notes. The values are the averages of the inner $\pm 105^{\prime \prime}$.

\subsection{Models}

To explain the aforementioned features in the velocity map and especially in the linewidth map and to determine the corrected rotation curve, an accurate model of NGC 4666 is essential. To model the data cube we used the FORTRAN code described in Kamphuis et al. (2007). In this code the emission is calculated

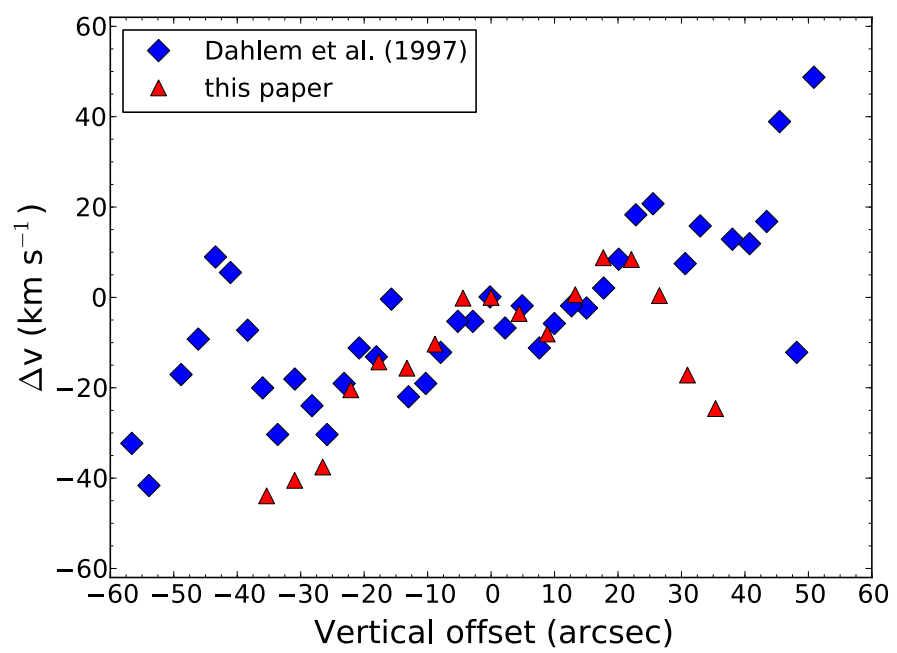

Fig. 5. Velocity offset along the minor axis of NGC 4666. The red triangles show our data; the blue diamonds show the data of Dahlem et al. (1997). For this comparison the sign of the vertical offset in the data of Dahlem et al. (1997) was switched because of the different convention of the position angle.

for every position in an exponential disk while taking the line of sight velocities into account.

The model takes the following characteristic parameters as input. The intrinsic shape of the ionized gaseous disk is characteristed by an exponential scale length and scale height, as well as a truncation radius. The velocity structure is determined from an input rotation curve and the given linewidth. Finally, the disk is orientated according to a given inclination.

The following parameters were determined directly from the data. The linewidth was measured from the diffuse gas in Fig. 2 as $35 \mathrm{~km} \mathrm{~s}^{-1}$. The initial rotation curve and inclination were taken from the output of ROTCUR. The truncation radius could be determined easily from the total flux map as $130^{\prime \prime}$, which corresponds to $11.5 \mathrm{kpc}$ assuming a distance of $18.2 \mathrm{Mpc}$ (see Table 1).

For the scale length and the scale height we started with some intitial estimates tracing the exponential intensity decrease along the major and minor axis, respectively. However, because of the large number of $\mathrm{H}_{\mathrm{II}}$ regions in the disk of NGC 4666, these values are very uncertain and had to be determined more accurately through a visual comparison of the data and the models.

As a first step in fitting the model to the data, we corrected the rotation curve and determined an accurate value for the scale length by comparing the PV-diagrams along the major axis of the data cube and the model cube to each other iteratively. This resulted in an exponential scale length of $3.0 \mathrm{kpc}$ and in corrected rotation velocities for the inner $15^{\prime \prime}$ (see Fig. 3). This correction is not unexpected as we did not perform an envelope tracing (Sofue \& Rubin 2001) to determine the initial rotation curve.

Subsequently, we determined accurate values for the inclination and the exponential scale height by calculating a grid of different models with different values for both parameters. This grid ranges from a scale height of $0.1 \mathrm{kpc}$ to $1.0 \mathrm{kpc}$ and from an inclination of $74^{\circ}$ to $82^{\circ}$. The resulting model cubes were analyzed in the same way as the data cube which resulted in a sample of radial velocity and linewidth maps, as seen in Figs. 6 and 7 , respectively.

We compared the models to the data through visual inspection of velocity fields and linewidth maps. This confirmed an 
P. Voigtländer et al.: The kinematics of the diffuse ionized gas in NGC 4666

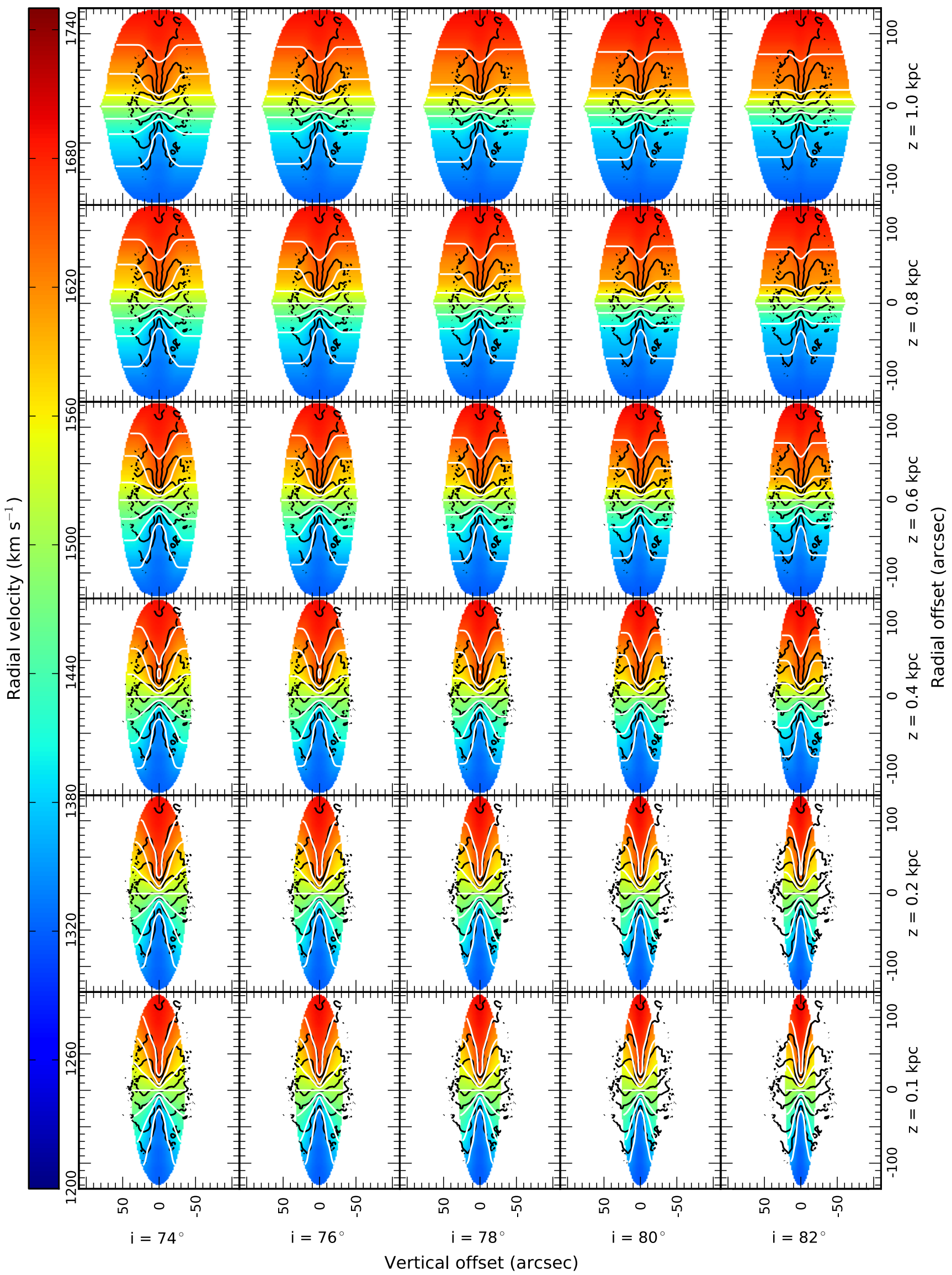

Fig. 6. Velocity maps of models with white contours in steps of $50 \mathrm{~km} \mathrm{~s}^{-1}$ around the systemic velocity of $1506 \mathrm{~km} \mathrm{~s}^{-1}$ at different inclination $(y$-axis) and scale heights ( $x$-axis). The black contours describe the data with the same velocity steps. 


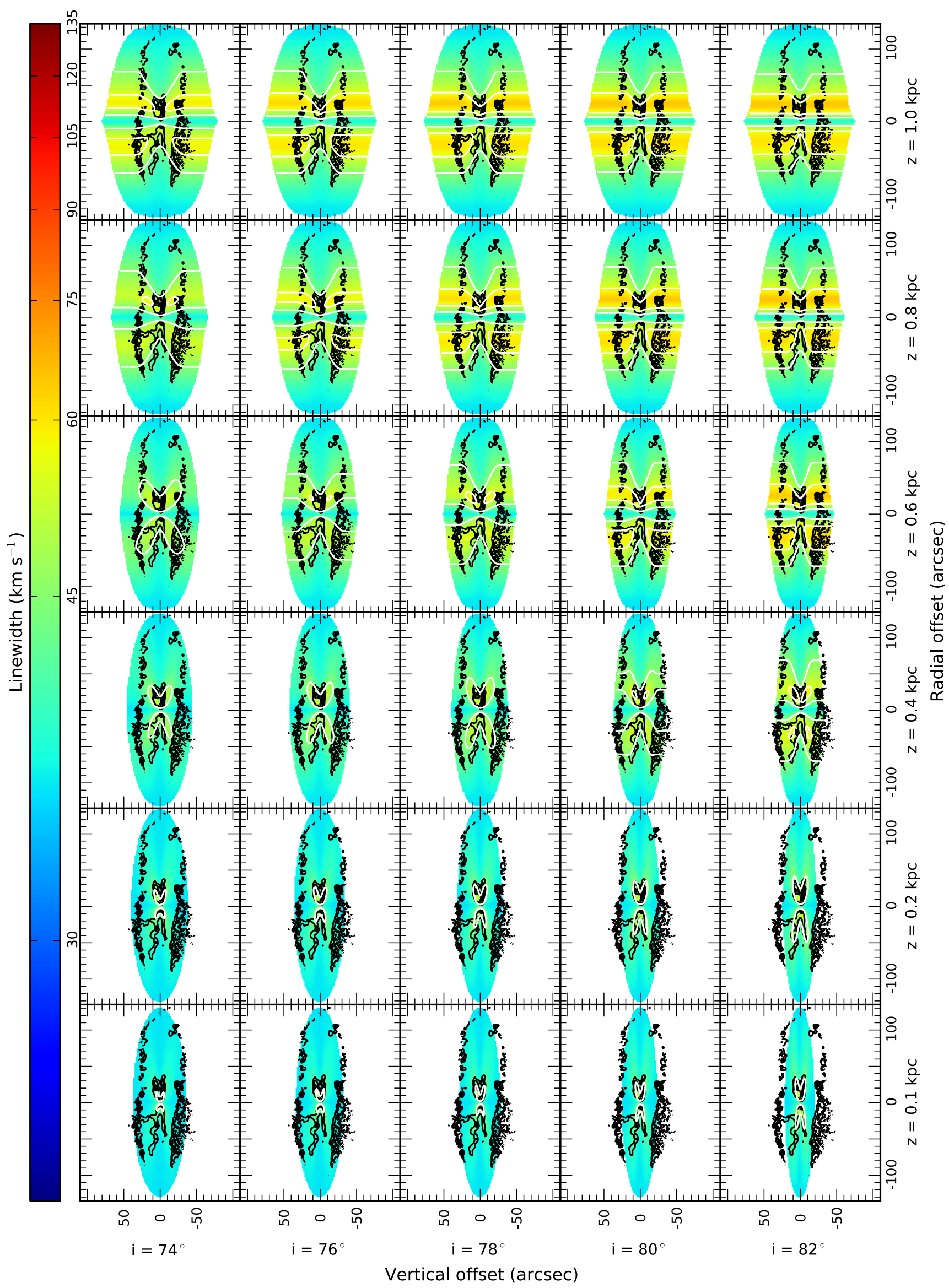

Fig. 7. Linewidth maps of models with white contours at $45 \mathrm{~km} \mathrm{~s}^{-1}, 55 \mathrm{~km} \mathrm{~s}^{-1}, 65 \mathrm{~km} \mathrm{~s}^{-1}$, and $75 \mathrm{~km} \mathrm{~s}^{-1}$ at different inclination ( $y$-axis) and scale heights ( $x$-axis). The black contours describe the data with the same linewidth steps. 


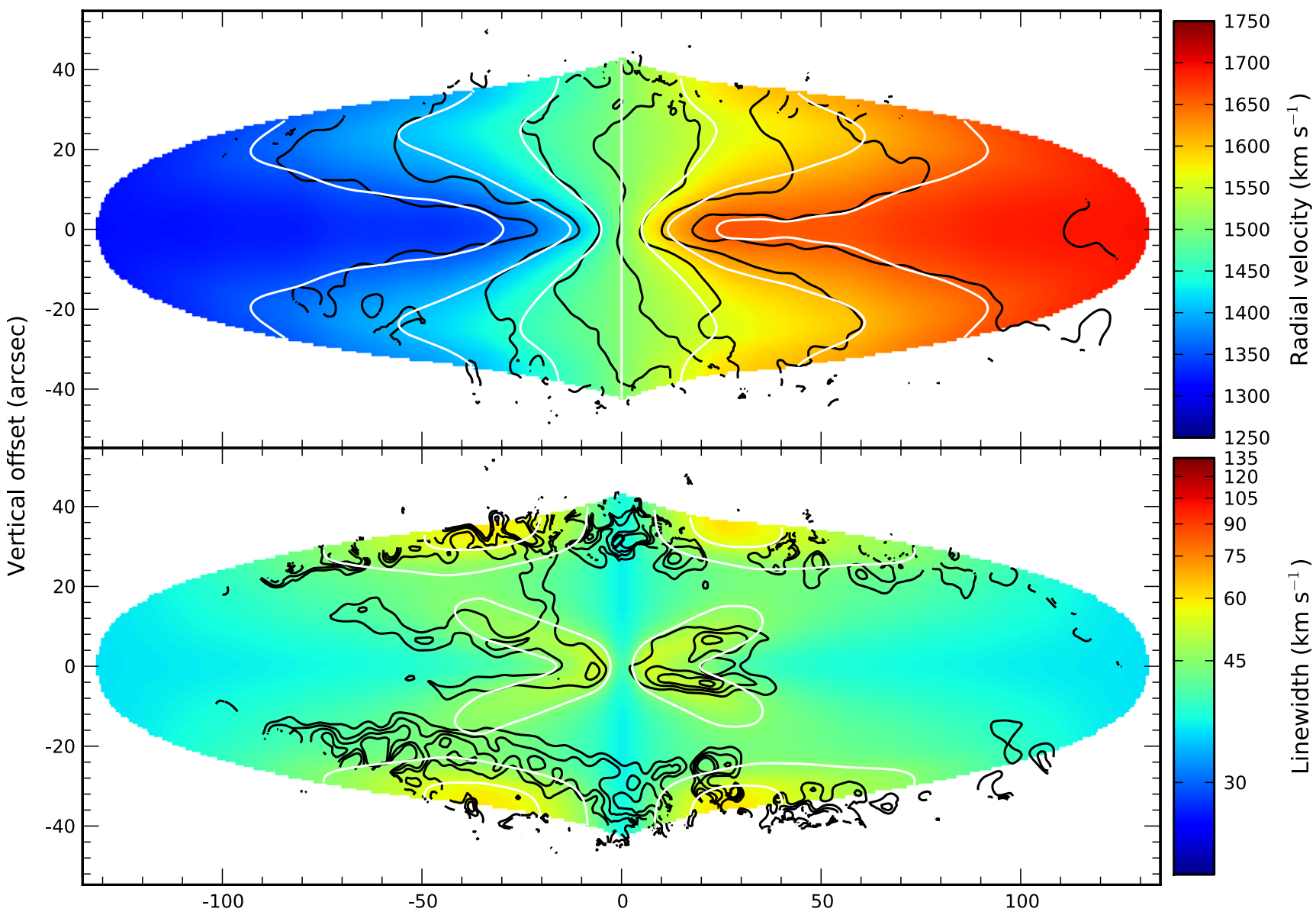

Radial offset (arcsec)

Fig. 8. Final velocity model (top) and the final linewidth model (bottom). The white contours show the model and the black contours the data. In the velocity map the contours are in steps of $50 \mathrm{~km} \mathrm{~s}^{-1}$ around the systemic velocity of $1506 \mathrm{~km} \mathrm{~s}^{-1}$. In the linewidth map the contours are at $45 \mathrm{~km} \mathrm{~s}^{-1}, 55 \mathrm{~km} \mathrm{~s}^{-1}, 65 \mathrm{~km} \mathrm{~s}^{-1}$, and $75 \mathrm{~km} \mathrm{~s}^{-1}$.

Table 3. Parameters for the best-fit model.

\begin{tabular}{ll}
\hline \hline Parameters (Unit) & Value \\
\hline Exponential scale length $(\mathrm{kpc})$ & 3.0 \\
Exponential scale height $(\mathrm{kpc})$ & $0.2 / 0.8$ \\
Inclination $\left(^{\circ}\right)$ & 78 \\
Truncation radius $(\mathrm{kpc})$ & 11.5 \\
Distance $(\mathrm{Mpc})$ & 18.2 \\
General linewidth $\left(\mathrm{km} \mathrm{s}^{-1}\right)$ & 35 \\
\hline
\end{tabular}

inclination of $78^{\circ}$ on the one hand and revealed a more complex structure of the scale height on the other hand. The inner part of the galaxy matched well with the model with a scale height of $0.2 \mathrm{kpc}$, whereas the outer part matched well with the model with a scale height of $0.8 \mathrm{kpc}$. Therefore, we tried a combination of these two models adding the thicker disk $(z=0.8 \mathrm{kpc})$ to the thin disk $(z=0.2 \mathrm{kpc})$. We found that a flux ratio between the thin disk and the thick disk of 2.5 matched the data best. A comparison of this final model with the data is shown in Fig. 8 for the velocity and linewidth map and confirms the quality of the calculated model. The parameters used in the final model are summarized in Table 3.

We also investigated models with a dust layer and a lagging halo to study the possible influences of such components. However, these models did not show any indication of the presence of a strong dust layer or a lagging halo.
In Fig. 8 the white contours describe the model and the black contours the data. In the velocity map (Fig. 8, top) one can see again offsets in velocity along the minor axis in the data. Otherwise, there is good agreement between model and data. All the residuals can be explained by the influence of $\mathrm{H}$ II regions except the upper-right quadrant, especially at a vertical offset of $20^{\prime \prime}$. The velocity in the data there is about $50 \mathrm{~km} \mathrm{~s}^{-1}$ higher than expected from the model. In comparison with our linewidth map (Fig. 2), one can see a small rise of the linewidth; however, it remains in good agreement with our model. A possible explanation of this velocity offset could be a warp.

In the linewidth map of the final model (Fig. 8, bottom) one can see an $x$-shaped structure in the central region with a maximum linewidth of $45 \mathrm{~km} \mathrm{~s}^{-1}$ whereas the linewidths in the outer parts show values of up to $60 \mathrm{~km} \mathrm{~s}^{-1}$. Compared to our data, the general structure is in good agreement. Nevertheless, there are two important differences. First, in our data the linewidth in the center rises to $75 \mathrm{~km} \mathrm{~s}^{-1}$ and is therefore $30 \mathrm{~km} \mathrm{~s}^{-1}$ higher than in the model. Second, the rising linewidth in the outer part is well described by the model for a positive radial offset; for a negative radial offset the linewidth rises to $120 \mathrm{~km} \mathrm{~s}^{-1}$, which is not described by the model. Increasing the inclination of the thin disk or thick disk does not solve this problem. We conclude that we are able to reproduce an increasing linewidth in an $x$-shaped structure in the center of the galaxy, but we are not able to reach the same strength. 


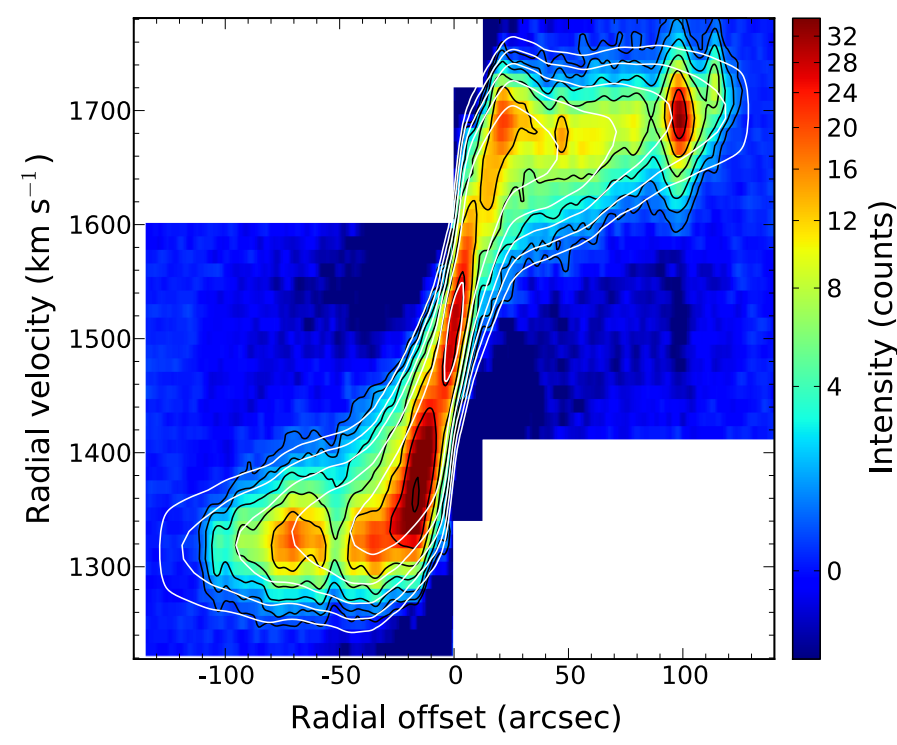

Fig. 9. PV-diagram of NGC 4666 along the major axis with the data in black and the final model in white contours.

To study the quality of our model Fig. 9 shows the PV-diagram along the major axis. The chosen scale length of $3.0 \mathrm{kpc}$ agrees well with the data, but the assumed dispersion of $35 \mathrm{~km} \mathrm{~s}^{-1}$ and the flat rotation velocity of $195 \mathrm{~km} \mathrm{~s}^{-1}$ also agrees well.

As a result we conclude that the regions of large linewidth in our data, marked by way of example with 3 and 4 in Fig. 2, are partly explained as projection effects of a thick and a thin disk in our data. For a complete explanation a detailed analysis and a comparison with other observations are necessary.

\subsection{Halo features}

To describe the halo features seen in Fig. 2 in more detail, a deeper view of the emission in the galaxy is necessary. For this we overlaid the $\mathrm{H} \alpha$ image from Dahlem et al. (1997) with our velocity image in contours in Fig. 10. To guide the eye, the black line representing a circle tilted at $78^{\circ}$ with a diameter of $130^{\prime \prime}$ is drawn as a rough approximation of the disk size.

First of all, we note that because of the lopsidedness of the galaxy the approximation of the disk with a simple ellipse fails on the left side. Furthermore, the above-mentioned influence of the $\mathrm{H}_{\mathrm{II}}$ regions in the velocity field is visible at several points, but more interesting are the structures below $-25^{\prime \prime}$ and above $+25^{\prime \prime}$ vertical offset. In the $\mathrm{H} \alpha$ image one can see extraplanar filaments reaching deep into the halo. In comparison to our velocity field one can see that our measurements go into these halo structures but are not as deep as the image by Dahlem et al. (1997). Nevertheless, a small protrusion in the velocity field at a radial and vertical offset of $30^{\prime \prime}$ also corresponds to an outgoing filament. The reasons that our observations do not show such structures in the halo are on the one hand the cut-off due to the ghost images which would influence the structure in the outer halo and, on the other hand the necessity to have a significant signal above the noise to realize a Gaussian fitting.

The structure of the galaxy in the lower-left quadrant is extremely interesting. In all other quadrants the ellipse describes the shape of the galactic disk fairly well, but not there. Between the ellipse and the real disk is a distance of about $10^{\prime \prime}$ which corresponds to $0.9 \mathrm{kpc}$. A possible explanation is the lopsidedness and, therefore, also a smaller size of the galactic height of the left part of the galaxy. However, in the linewidth map (Fig. 2) this region shows a strong increase of the linewidth that cannot be explained with our models. Furthermore, in Fig. 10 several filamentary structures and knots of higher emission like in the hook-like structure close to position 5 are visible. Therefore, these structures could represent outflowing extraplanar gas.

The behavior of the velocity along the minor axis, especially the double Gaussian structure at region 2, can be understood better when taking into account the filamentary structures above $+25^{\prime \prime}$ vertical offset that can be seen in the $\mathrm{H} \alpha$ image. We interpret this gas as part of an outflow. In region 6 no H II region is visible, but strong emission of DIG up to a vertical offset of $-50^{\prime \prime}$. Therefore, an overlap of the normal rotation velocity and the velocity of the outflowing gas can be expected there.

\section{Comparison with observations in other wavelengths}

For the final interpretation a comparison with observations in other wavelengths is necessary.

Dahlem et al. (1997, 1998) detected extraplanar soft X-ray emission and radio synchrotron emission from the halo and interpreted these as further evidences for a galactic outflow. Walter et al. (2004) detected H i tidal streams connecting to NGC 4668. Furthermore, they assumed that the high SFR is triggered by gravitational interaction of NGC 4666 with the other galaxies in the group. For the $\mathrm{H}_{\mathrm{I}}$ Walter et al. (2004) determined an inclination for the inner $2^{\prime}$ of about $70^{\circ}$ whereas our determination of the inclination amounts to $78^{\circ}$. This results in a velocity offset of about $7 \mathrm{~km} \mathrm{~s}^{-1}$. Taking this into account the maximal rotation velocity in Walter et al. (2004) is $173 \mathrm{~km} \mathrm{~s}^{-1}$. This is in good agreement to the value of the inner arcminute in our analysis of the ionized hydrogen and still in agreement to our maximal rotation velocity of $195 \mathrm{~km} \mathrm{~s}^{-1}$ when taking into account the errors.

Although optical observations, including our own observation, do not show any evidence of a strong interaction with other galaxies, such as a tidal streams, NGC 4666 cannot be seen as an isolated galaxy. Especially the lopsidedness of NGC 4666 could be a hint for such a gravitational interaction with other galaxies. It is unlikely that the regular thick gaseous disk found in this study would be caused by environmental effects. But finally one has to take into account also the surrounding galaxies for a complete interpretation. However, the data for such an analysis is currently not available.

\section{Summary and conclusions}

We observed NGC 4666, an edge-on galaxy with a high SFR, with a Fabry-Pérot interferometer to get line of sight velocity information of the ionized hydrogen in the disk and in the halo. Based on these data we calculated the rotation velocities, which result in a flat rotation curve with a rotation velocity of about $195 \mathrm{~km} \mathrm{~s}^{-1}$.

A comparison of the velocity along the minor axis, which should be constant, with the results of Dahlem et al. (1997) yields a good agreement within the inner $\pm 20^{\prime \prime}$ of vertical offset. The differences at higher vertical offsets can be attributed to additional velocity components, which are present in our data and not in the data of Dahlem et al. (1997) as a result of our better spectral resolution. Especially for a positive vertical offset of more than $20^{\prime \prime}$, a second velocity component with a velocity 


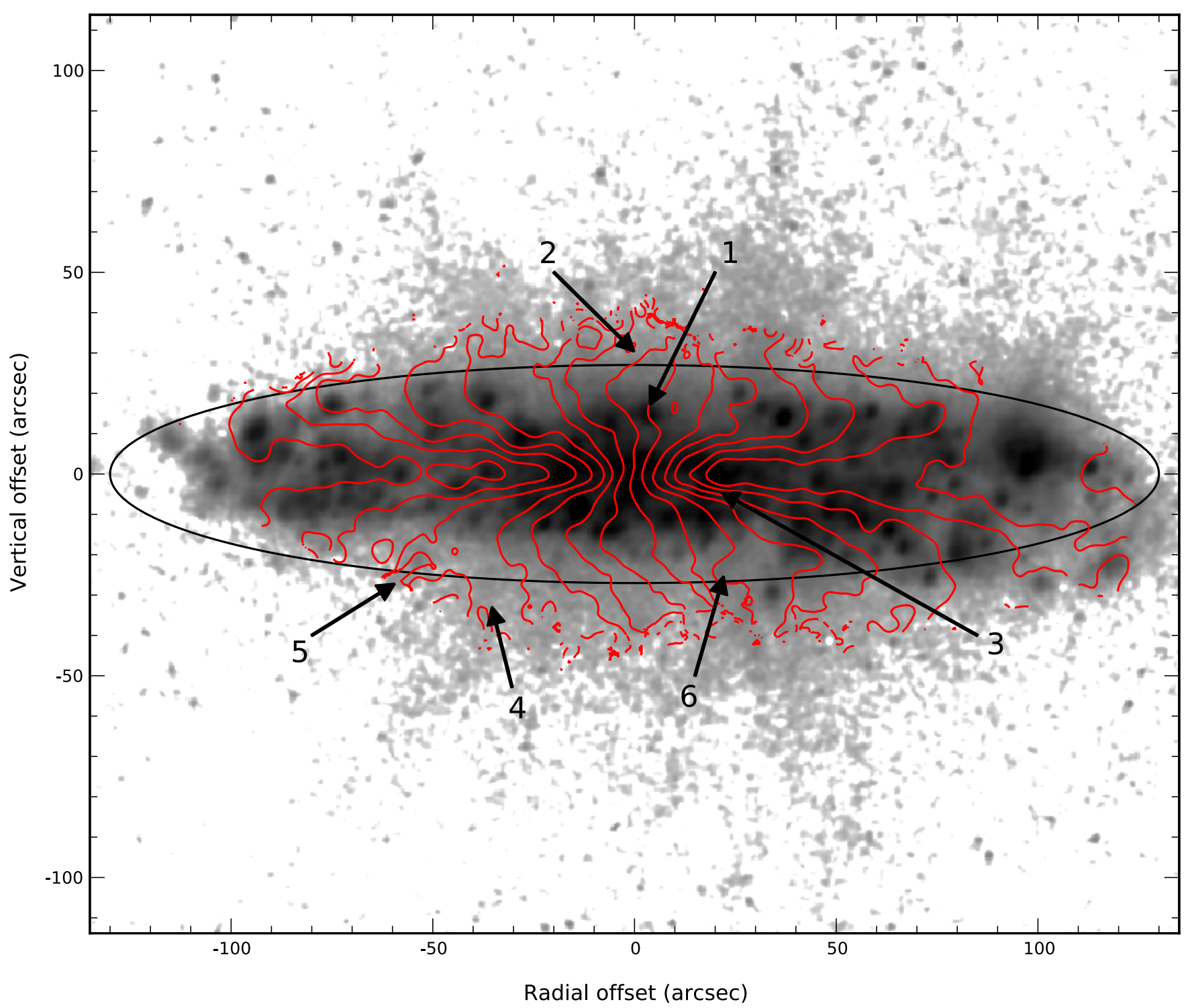

Fig. 10. $\mathrm{H} \alpha$ from Dahlem et al. (1997) overlaid with the velocity contours of our data in steps of $25 \mathrm{~km} \mathrm{~s}^{-1}$ around the systemic velocity of $1506 \mathrm{~km} \mathrm{~s}^{-1}$ and a rough approximation of the disk size by tilting a circle with a diameter of $130^{\prime \prime}$ to the inclination of the galaxy $\left(78^{\circ}\right)$.

offset of about $-100 \mathrm{~km} \mathrm{~s}^{-1}$ is confirmed, which we interpret as a strong outflow.

To interpret the velocity and the linewidth of the fitted profiles in an appropriate way we created models with different parameters like inclination and scale height, based on the determined rotation velocity. As result of comparing these different models with the data, a combination of two models, one with a thin disk $(z=0.2 \mathrm{kpc})$ and one with a thick disk $(z=0.8 \mathrm{kpc})$, gave the best fit. A first important result is that the double Gaussian $x$-shaped structure in the center of the linewidth map as well as the double Gaussian structure in the outer regions at negative radial offsets are results of projection effects. The exact shape and the linewidth differs on small scales between data and model, but because of the large number of $\mathrm{H}$ II regions embedded in the DIG of the disk this is not surprising. We conclude as the main result of this paper the existence of a thin and a thick disk of DIG in NGC 4666, similar to the one in NGC 891 (Dettmar 1990; Rand et al. 1990) or in our own Galaxy (Reynolds 1990). Finally, we can conclude that modeling the linewidth map offers a proper possibility of detecting a thin and a thick disk of ionized gas in highly inclined, but not edge-on, galaxies.

Furthermore, we found extraplanar structures, e.g., at a radial offset of $25^{\prime \prime}$ in both vertical directions, consistent with previous $\mathrm{H} \alpha$ observations. Clearly, the star formation in NGC 4666 maintains a diffuse ionized thick disk and drives also a large scale outflow. If there is really a galactic superwind, cannot be concluded with our data. It is worth noting here that the outflow appears driven by a high star forming disk and not a nuclear starburst. This makes NGC 4666 more similar to $z=2$ turbulent disks (e.g., Genzel et al. 2011) than merger driven nuclear starbursts (e.g., Heckman et al. 1990).

Acknowledgements. This research has made use of the NED, which is operated by the Jet Propulsion Laboratory, California Institute of Technology, under contract with NASA. We acknowledge the use of the HyperLeda data base (http://leda. univ-lyon1. fr). We thank J. L. Gach, P. Balard, and O. Boissin for preparing the instrument for the observations and for repairing a failure in the cooling system during the run. P. Kamphuis thanks the Alexander von Humboldt Foundation for financial support during his stay in Germany. Research in this area at Ruhr-Universität Bochum is supported by FOR 1048. Last but not least, we want to thank the referee for useful comments. 


\section{References}

Buat, V., \& Xu, C. 1996, A\&A, 306, 61

Dahlem, M., Petr, M. G., Lehnert, M. D., Heckman, T. M., \& Ehle, M. 1997, A\&A, 320, 731

Dahlem, M., Weaver, K. A., \& Heckman, T. M. 1998, ApJS, 118, 401

Daigle, O., Carignan, C., Hernandez, O., Chemin, L., \& Amram, P. 2006, MNRAS, 368, 1016

Dettmar, R.-J. 1990, A\&A, 232, L15

Dettmar, R. J. 1992, Fund. Cosmic Phys., 15, 143

Epinat, B. 2009, Ph.D. Thesis, Université de Province, France

Fitzpatrick, E. L. 1999, PASP, 111, 63

Fraternali, F., Oosterloo, T. A., Sancisi, R., \& Swaters, R. 2005, in Extra-Planar Gas, ed. R. Braun, ASP Conf. Ser., 331, 239

Gach, J.-L., Hernandez, O., Boulesteix, J., et al. 2002, PASP, 114, 1043

Garcia, A. M. 1993, A\&AS, 100, 47

Genzel, R., Newman, S., Jones, T., et al. 2011, ApJ, 733, 101

Gooch, R. 1996, in Astronomical Data Analysis Software and Systems V, eds. G. H. Jacoby, \& J. Barnes, ASP Conf. Ser., 101, 80

Heald, G. H., Rand, R. J., Benjamin, R. A., \& Bershady, M. A. 2006a, ApJ, 647, 1018

Heald, G. H., Rand, R. J., Benjamin, R. A., Collins, J. A., \& Bland-Hawthorn, J. 2006b, ApJ, 636, 181

Heckman, T. M. 2002, in Extragalactic Gas at Low Redshift, eds. J. S. Mulchaey, \& J. T. Stocke, ASP Conf. Ser., 254, 292

Heckman, T. M., Armus, L., \& Miley, G. K. 1990, ApJS, 74, 833

Kamphuis, P., Peletier, R. F., Dettmar, R.-J., et al. 2007, A\&A, 468, 951

Kennicutt, R. C., Jr. 1998, ARA\&A, 36, 189

Kennicutt, R. C., Jr., Hao, C.-N., Calzetti, D., et al. 2009, ApJ, 703, 1672

Lehnert, M. D., \& Heckman, T. M. 1995, ApJS, 97, 89

Lehnert, M. D., \& Heckman, T. M. 1996a, ApJ, 462, 651
Lehnert, M. D., \& Heckman, T. M. 1996b, ApJ, 472, 546

Marinacci, F., Fraternali, F., Nipoti, C., et al. 2011, MNRAS, 415, 1534

Martin, C. L., Kobulnicky, H. A., \& Heckman, T. M. 2002, ApJ, 574, 663

Moustakas, J., \& Kennicutt, R. C., Jr. 2006, ApJS, 164, 81

Norman, C. A., \& Ikeuchi, S. 1989, ApJ, 345, 372

Oosterloo, T., Fraternali, F., \& Sancisi, R. 2007, AJ, 134, 1019

Osterbrock, D. E. 1989, Astrophysics of gaseous nebulae and active galactic nuclei

Rand, R. J. 1996, ApJ, 462, 712

Rand, R. J., Kulkarni, S. R., \& Hester, J. J. 1990, ApJ, 352, L1

Reynolds, R. J. 1990, in The Galactic and Extragalactic Background Radiation, eds. S. Bowyer, \& C. Leinert, IAU Symp., 139, 157

Rossa, J., \& Dettmar, R.-J. 2000, A\&A, 359, 433

Rossa, J., \& Dettmar, R.-J. 2003a, A\&A, 406, 493

Rossa, J., \& Dettmar, R.-J. 2003b, A\&A, 406, 505

Shapiro, P. R., \& Field, G. B. 1976, ApJ, 205, 762

Shields, G. A. 2002, in Rev. Mex. Astron. Astrofis. Conf. Ser. 12, eds. W. J. Henney, J. Franco, \& M. Martos, 189

Sofue, Y., \& Rubin, V. 2001, ARA\&A, 39, 137

Soifer, B. T., Boehmer, L., Neugebauer, G., \& Sanders, D. B. 1989, AJ, 98, 766 Springob, C. M., Masters, K. L., Haynes, M. P., Giovanelli, R., \& Marinoni, C. 2007, ApJS, 172, 599

Tomisaka, K., Habe, A., \& Ikeuchi, S. 1981, Ap\&SS, 78, 273

van der Hulst, J. M., Terlouw, J. P., Begeman, K. G., Zwitser, W., \& Roelfsema, P. R. 1992, in Astronomical Data Analysis Software and Systems I, eds. D. M. Worrall, C. Biemesderfer, \& J. Barnes, ASP Conf. Ser., 25, 131

van Eymeren, J., Marcelin, M., Koribalski, B., et al. 2009a, A\&A, 493, 511

van Eymeren, J., Marcelin, M., Koribalski, B. S., et al. 2009b, A\&A, 505, 105

Veilleux, S., Cecil, G., \& Bland-Hawthorn, J. 2005, ARA\&A, 43, 769

Walter, F., Dahlem, M., \& Lisenfeld, U. 2004, ApJ, 606, 258

Xiao, T., Wang, T., Wang, H., et al. 2012, MNRAS, 421, 486 